\title{
Ethical Issues in Intensive Care Units during the COVID-19 Pandemic
}

\author{
Vasileios Karampelias ${ }^{1}$, Ypatios Spanidis², Elpida Roussakou ${ }^{3}$
}

\begin{abstract}
Coronavirus disease-2019 (COVID-19) outbreak began in December 2019 in China and has spread rapidly across the world. The healthcare system of each country has been affected from this situation. Undoubtedly, during this period several ethical issues have been raised. In this commentary, we aimed to make a discussion regarding the ethical issues that could be raised in the treatment of patients in the intensive care units during the COVID-19 pandemic. The objective of this article is to contribute to the wide current discussion regarding the appropriate measures that should be taken to protect the health and ensure the safety of the staff that comes in close contact with patients who are suspected or confirmed of having COVID-19.
\end{abstract}

Keywords: Ethical issues, Intensive care unit, Surgical intensive care unit.

Indian Journal of Critical Care Medicine (2020): 10.5005/jp-journals-10071-23543

Coronavirus disease-2019 (COVID-19) outbreak that began in December 2019 in China has spread rapidly across the world, infecting more than 2.5 million people and has resulted in approximately 165,000 deaths near the end of April 2020.' The global communities are facing the largest crisis in the last decades, with the most rapid and radical changes in social life in the Western world. The economies and markets of most countries are in lockdown, and healthcare services have been profoundly affected.

The healthcare system of each country is in the frontline of this battle. Many patients are admitting every day in intensive care units (ICUs) with suspected or confirmed COVID-19, and many of them require immediate care, which has become the priority and urgent preoccupation of the medical staff. The physicians are fighting to save patient lives and prevent the transmission of the virus by exposing themselves to the risk of infection, sometimes with limited human and equipment resources (e.g., ventilators, hospital beds, personal protective equipment). It should be noted that even the Italian healthcare system, which is one of the most regarded in Europe with 3.2 beds per 1,000 people (the corresponding rate in the United States is 2.8 per 1,000 people), appears to be unable to provide health care to the increasing number of simultaneously critically ill patients. ${ }^{2}$

Therefore, each government should develop a clear strategy to protect the healthcare workers, manage the available resources, and provide the best possible level of health care to the patients. Clearly, the infection of the medical staff and their subsequent self-isolation would be a blow to this effort. The example of Spain is striking, as approximately $14 \%$ of the infected population comprises healthcare workers. ${ }^{3}$ Indeed, of all hospital departments, the personnel in ICUs are possibly those who experience the greatest pressure and stress in providing care for patients with severe health problems, many of whom hold diverse cultural beliefs, values, and attitudes around serious illness and death. ${ }^{4}$ As a result, the ICU personnel must be on high alert for rapid clinical management and infection prevention measures to ensure the health of healthcare workers and patients not directly affected by COVID-19 who are at risk of nosocomial transmission or in need of ICU admission for unrelated reasons. ${ }^{5}$

\author{
1,2Department of Surgery, School of Medicine, University of Patras, \\ Patras, Greece \\ ${ }^{3}$ Mediterranean College, Athens, Greece \\ Corresponding Author: Ypatios Spanidis, Department of Surgery, \\ School of Medicine, University of Patras, Patras, Greece, Phone: \\ +306980068997, e-mail:akis.span@yahoo.gr \\ How to cite this article: Karampelias V, Spanidis Y, Roussakou E. Ethical \\ Issues in Intensive Care Units during the COVID-19 Pandemic. Indian J \\ Crit Care Med 2020;24(9):855-856. \\ Source of support: Nil \\ Conflict of interest: None
}

However, the development of prevention measures may include crucial decisions that raise ethical issues. Specifically, the increased number of patients admitted to the ICUs has led to the evaluation of each case and the management of each patient according to his/her condition. Indeed, those with respiratory failure, multiple organ dysfunction, and dyspnea are classified as critical and require immediate admission to the ICU. Critically ill patients with COVID19 tend to be older ( $>65$ years) and may have a larger number of underlying conditions. ${ }^{2}$ Especially in Italy, Spain, and the United States, which are among the most affected countries from the pandemic, the limited availability of ventilators and hospital beds compared to the increasing number of cases has led to the setting of an age limit regarding the provision of ventilation support to the patient. ${ }^{2}$ In line, the decision for providing ventilation support or any form of treatment to a patient with COVID-19 may derive after considering and evaluating the presence of comorbidities, the severity of respiratory failure, and the estimated time of intubation. Actually, the physicians in many healthcare facilities appear to value most saving patients who have the greatest chance to survive over those who do not. It has become clear that offering a ventilator to a patient who is extremely unlikely to survive denies the opportunity for ventilator support to another patient with greatest chances of survival. ${ }^{2}$ Therefore, in many cases, the physicians have to decide

(c) The Author(s). 2020 Open Access This article is distributed under the terms of the Creative Commons Attribution 4.0 International License (https://creativecommons. org/licenses/by-nc/4.0/), which permits unrestricted use, distribution, and non-commercial reproduction in any medium, provided you give appropriate credit to the original author(s) and the source, provide a link to the Creative Commons license, and indicate if changes were made. The Creative Commons Public Domain Dedication waiver (http://creativecommons.org/publicdomain/zero/1.0/) applies to the data made available in this article, unless otherwise stated. 
who would die and who would survive, a dilemma similar to those faced during wartime. ${ }^{2}$ Nevertheless, the criterion of severity of the affected patient is not sufficient, as COVID-19 presents rapid recrudescence.

Likewise, Italy and China first suggested the cancelation and postponement of elective and semi-elective surgeries, respectively, during the pandemic to reduce the danger of infection for the surgical staff, enhance the available human resources in the ICUs, and even turn the operating rooms into makeshift ICUs. ${ }^{2,5}$ Although this measure is considered mandatory and has been adopted by many other countries, it may hide the risk of delaying an elective operation for a chronic disease, such as cancer, limb salvage, or a benign disease with a risk of infection, which would lead to delayed treatment outcomes and may worsen the patient's condition. ${ }^{5}$

To more effectively solve such ethical dilemmas, the personnel in the ICUs caring for patients have to be sufficient in numbers and be physically and psychologically supported by the administration. The medical staff should be well-informed and trained regarding the procedures and the decisions that need to be made. Moreover, the workers in the ICUs should seek advice from more experienced healthcare workers when crucial decisions must be made. For this reason, the hospitals, and especially the ICUs, should be connected via telephone and other online technologies to exchange information. Cooperation among medical staff could lead to better prognosis, evaluation, and treatment decision for patients with COVID-19, thus enhancing their chances of survival.

To do so, it is important to separate clinicians who provide care from those who make decisions. Specifically, a model that has been proposed and adopted in Italy suggests the presence of an "officer," backed by a team of experts in nursing and respiratory therapy, who are responsible for resource-allocation decisions and the communication with the patients and the family members. These decisions are reviewed by a state-level committee to ensure equal distribution of resources and effectively manage the availability of the resources in this area. The review of these data could be also used as knowledge regarding disease evolution. ${ }^{2}$
Considering the aforementioned ethical issues, it becomes clear that the healthcare systems and workers are presently unable to provide the fundamental Hippocratic ethical principles of beneficence, non-maleficence, justice, and respect of autonomy in the ICUs during the COVID-19 pandemic. The need for developing specific protocols regarding the management of patients and the training of personnel is highlighted as we go through the pandemic. As there is only a thin line between preparedness and panic, the trust between patients and healthcare providers may be the key point to achieving ideal cooperation and increasing the possibilities for the effective treatment and cure of as many patients as possible.

\section{ACKnowledgments}

We would like to thank "The Sciencing Team" (https:// thesciencingteam.com) for the thorough English editing of our manuscript and for publication support.

\section{References}

1. Lai CC, Shih TP, Ko WC, Tang HJ, Hsueh PR. Severe acute respiratory syndrome coronavirus 2 (SARS-CoV-2) and coronavirus disease-2019 (COVID-19): the epidemic and the challenges. Int J Antimicrob Agents 2020;53(3):105924.

2. Rosenbaum L. Facing COVID-19 in Italy-ethics, logistics, and therapeutics on the epidemic's front line. N Engl J Med 2020;382(20):1873-1875. DOI: 10.1056/NEJMp2005492.

3. Brindle M, Gawande A. Managing COVID-19 in surgical systems. Ann Surg 2020;272(1):e1-e2. DOI: 10.1097/SLA.0000000000003923.

4. Phua J, Weng L, Ling L, Egi M, Lim C-M, Divatia JV, et al. Intensive care management of coronavirus disease 2019 (COVID-19): challenges and recommendations. Lancet Respir Med 2020;8(5):506-517. DOI: 10.1016/S2213-2600(20)30161-2.

5. Chew MH, Koh FH, Ng KH. A call to arms: a perspective of safe general surgery in Singapore during the COVID-19 pandemic. Singapore Med J 2020;61(7):378-380. DOI: 10.11622/smedj.2020049. 